Interactive comment on "Assessing and improving cloud-height based parameterisations of global lightning flash rate, and their impact on lightning-produced $\mathrm{NO}_{x}$ and tropospheric composition" by Ashok K. Luhar et al.

Anonymous Referee \#3

Received and published: 5 October 2020

Section 3.1

In line 15 of page 9, it is said "... by substituting Eq. (8) into Eq(13) ...", wouldn't it be the opposite?.

Section 3.5

According to the authors ACCESS-UKCA was setup as a free running simulation for 2 years (2005 and 2006), and the simulation was started using the model initial conditions taken from a nudged model run (see line 10 in page 13). I think the authors should 
be a bit more specific on this technical matter. I underdtand that the nudging somehow guarantees / ensures that the basic dynamics in the lower-middle atmosphere is identical in simulations in which other changes (implementation of a lightning scheme for instance) are made. Is the nudging applied to all altitudes (pressure levels of the model)?. Also, it is not clearly indicated whether lightning was included (or not) in the first free running simulation. Was it?.

Interactive

comment

I consider that to "see" the influence of lightning only in a CTM one should proceed as the following: First run your code in a free-running dynamic mode without considering lightning. Then run a second model simulation also without lightning but now using the nudge, that is, the horizontal wind and temperature fields in the tropo-stratosphere are nudged at each model time step of the first free-running dynamic ACCESS-UKCA run. Then, what I would do, is to run a third nudged ACCESS-UKCA simulation (with the lightning scheme on) that is nudged to the first free-running dynamics ACCESS-UKCA run. Finally, I would repeat the third simulation for each of your lightning schemes (PR92 and your new one) and will always compare their output with the results of the second nudged ACCESS-UKCA runs. In this way you will ensure that you are really carrying out comparisons between simulations of the atmosphere with and without lightning that are not biased by dynamical effects.

It is not completely clear to me if your "nudged model run" is really free of dynamical effects. Please comment on this and try to be more specific.

\section{Section 3.6}

As a remark, by looking at Table 1 I see that the output of RUN 1 (PR92) gives quite low global lightning flash frequency (32.92 flashes / s). Note that the UKCA-UM model was already used by Finney et al 2016 and applied a scaling factor of 1.44 to match PR92 global flash frequency to LIS/OTD observations. In your case the scaling factor would be 1.40 .

Printer-friendly version

Discussion paper

Could you please explain a bit the underlying reasons for your model runs (including 
RUN 2) to underpredict in spring in the $\mathrm{SH}$ and $\mathrm{NH}$ and overpredict in autumn in the $\mathrm{SH}$ (see Figures $3 \mathrm{a} / \mathrm{b}$ ) ?.

Both PR92 and the new lightning scheme proposed fail in accurately describing the tropical oceanic flash rate (see Fig. 4c). There is a considerable overestimation of RUN 1 (PR92) and RUN 2 (new lightning scheme). What is the reason for this?. This has consequences on the simulation results shown in Fig. 5a (observations) and Fig. 5d (new scheme) where the tropical oceanic overestimated flash rate is apparent. Please comment a bit on this behaviour.

Regarding land, note that North America, the Indian and Australian continents are not very much well described either in RUN 1 and RUN 2 (new scheme). Please give reasons for this.

In commenting the use of scaling factor for flash frequency (line 1 and 2 of page 20) you should also cite the works by Tost et al 2007, Finney et al 2016 and Clark et al 2017 (among others) that applied such scaling factors in different models.

Regarding scaling for NO produced per flash, authors have prescribed an amount of 330 moles NO/flash which immediately conditions the desired lightning generated NOx (LNOx) as can be clearly seen from equation (21). Any comment on this?.

The authors are assuming that all lightning flashes produce 330 moles NO / flash (no matter if CG or IC and independently of occurring in land or ocean). However, it is known that CG strokes over water usually carry more charge into them which leads to a higher transported current. This is an indication that, on average, CGs over water are more energetic than CGs over land and, consequently, CGs over ocean would produce a larger LNOx (see the paper by Nag and Cummings in GRL 2017). The latter is an indication of different land / ocean convection regimes. This is not considered by any lightning scheme (quantifiying the occurrence rate, not the energy). Authors should add comments on these deficiencies so that readers can have a fair perspective of the many limitations of lightning schemes (any). 


\section{Section 3.7}

\section{Subsection 3.7.1}

In my view the lack of scaling flash frequencies (and the fact of using a prescribed $\mathrm{P} \_\mathrm{NO}=330$ moles NO per flash) artificially magnifies the difference between the PR92 $\mathrm{LN} O x$ (4.8 Tg N / yr) and the one resulting from the new lightning scheme (RUN 2) leading to $6.6 \mathrm{Tg} \mathrm{N} / \mathrm{yr}$. If authors would have scaled (to match observations) the flash frequencies of each tested lightning scheme (especially the one of PR92), the resulting LNOx of PR92 and TS1 would have been much closer.

In connection with this, I miss a deep discussion on the reasons for selecting 330 moles $\mathrm{NO} /$ flash. For example, there are recent papers (not cited by the authors) by Bucsela et al JGR-Atm 2019 and Allen et al JGR-Atm 2019 where, based on OMI + WWLLN observations, find that LNOx can be 180 moles NO / flash +- 100 in midlatitudes summertime NH. Complementarily, the paper by Allen et al 2019 finds that LNOx can range between 70 and 270 moles $\mathrm{NO} /$ flash in the tropics.

I disagree with the sentence in lines 11-12 of page 22 that the new flash-rate parameterization (Fig. 6b) agrees better with annual LNOx distribution obtained by Miyazaki et al 2014 (Fig. 6c). There are large land geographical regions (North America, Australia, India, EuroAsia) where the predicted LNOx by PR92 and the new scheme are pretty similar and very different with respect the LNOx distribution derived by Miyazaki et al 2014 (Fig. 6c). This is mainly due to the very different flash densities of both PR92 and RUN 2 (Fig. 5c and 5d) compared to observations (Fig. 5a). So, the global flash frequency (and LNOx) could be similar to considered observations (LIS / OTD and Miyazaki's 2014) but, to me, a more demanding comparison would require detailed comparison of flash frequencies (and LNOx) per continental region (North America, South America, Africa, EuroAsia, ...). Could the authors provide a Table showing such comparison?. 
The vertical distribution of LNOx is crucial. The authors compare their chosen vertical distribution with those of Pickering et al 1998 and Ott et al 2010. The paper adopts an alternative vertical distribution closer to Ott's. However I miss a full discussion explaining / supporting the reasons that moved the authors to use the vertical LNOx distribution (blue dots) shown in Fig. 7.

Interactive

Please comment and justify your election of vertical distribution. Do you have supportive observations?. Why do you use these profiles?.

The relative energy of global ICs with respect to global CGs has consequences and / or conditions the LNOx vertical distributions. For instance, the vertical LNOx introduced by Pickering et al 1998 is consistent with their election of IC flashes being 10 $\%$ as energetic as CG flashes. In fact, according to Pickering et al 1998, if global IC flashes contained less than $10 \%$ energy as CG flashes, the upper troposphere (UT) peak (upper part of the "C-shaped" distributions) in the mass profiles might not be as pronounced. Consequently, if ICs are equally energetic as CGs (as authors have assumed) the UT peak would be even more significant and this is not consistent with the vertical distribution used by the authors that rather seems to be a kind of mean between Pickering's and Ott's distribution. But, again, what are your physical / chemical / transport reasons supporting such profiles?.

\section{Subsection 3.7.3}

While I understand the authors' reasoning for tropospheric NO2 verification, I do not fully agree with your conclusions of this section.

As I see it, the conflict in your procedure starts in line 6 of page 25 . Here you indicate that since N_v_trop_180 is not available from observations, you take the average of the curves (in Figure 8) showing the predicted N_v_trop_180, that is, the mean tropospheric NO2 column vs latitude resulting from RUN 1 (PR92) and RUN 2 (new scheme) over the reference longitude of 180 degrees in 2006 . Doing this somehow "contaminates" the reference, that is, the CAMS data. This "contamination" leads to curves like

Printer-friendly version

Discussion paper 
the ones shown in Fig. 9 where, inevitably, RUN 1 and RUN 2 for global, land and oceanic scenarios are strangely close to the CAMS values (considered as reference).

Do Fig. 9 show total NO2 columns or only the lightning contribution to the zonal annualmean tropospheric NO2 column?. If total, please state it clearly.

I miss comparison of your NO2 values (shown in Fig. 9) with NO2 values reported in Bucsela et al 2019 (see Fig. 3(a) there) from OMI + WWLLN observations in northern midlatitude regions.

Please elaborate on this a bit.

Section 4 / Impact on chemical tropospheric composition

Let me start by indicating that in this section I miss a more detailed discussion on explicit chemical reactions and species in the context of the production / loss of the lightning affected species ( $\mathrm{NOx}, \mathrm{O} 3, \mathrm{OH}$ and $\mathrm{CO}$ ) in the different geopraphical regions. As mentioned in line 32 of section 2, the model includes 306 chemical reactions and 86 species. This chemical set (plus the aerosol chemistry) is quite rich so that key chemical processes could have been pointed out. This is not really done.

Please try to indicate the key processes that, according to the model's reaction set, play the most important role(s) for the formation / loss of each of the species investigated. This is very important and illuminating for the readers.

\section{Section 4.1 (NOx)}

As said above I think that the comparison between modelled tropospheric NO2 columns and observations shown in section 3.7.3 is not completely convincing.

Here you compare the total tropospheric NO2 colums resulting from PR92 and the new lightning scheme and its difference. I think it would have been clearer for readers to show only the corresponding lightning contributions to the tropospheric NO2 column.

Section $4.2(\mathrm{O} 3)$ 
Could you please indicate the explicit chemical mechanisms that (according to the adopted chemical set) are controling the balance of $\mathrm{O} 3$ at $20 \mathrm{~m}$ and at $6400 \mathrm{~m}$ due to lightning activity?. What are the key chemical processes controlling ozone population at the two considered reference altitudes?. Are they the same or different?. This is an interesting information not commented in the paper.

Why, according to the authors, the new lightning scheme is not really able to account for the $\mathrm{O} 3$ observations in Fig. 12(c) and Fig. 12 (e)?.

\section{Section $4.3(\mathrm{OH})$}

Could you please show only the lightning contribution to the total $\mathrm{OH}$ tropospheric column ?. It is also important to show readers what are the crucial chemical reactions due to the increase of $\mathrm{OH}$ at $20 \mathrm{~m}$ and $6400 \mathrm{~m}$.

The authors openly admit that the UKCA StratTop configuration produces an overestimation of $\mathrm{OH}$. It would be interesting for readers if the authors could dig into their chemical scheme and indicate what chemical processes could be playing a role (or could somehow explain) the modelled overestimation.

\section{Please comment.}

\section{Section 4.4 (CO)}

Are the authors showing in Fig. 17 the total annual-mean tropospheric $\mathrm{CO}$ or only the one due to lightning?.

\section{Recommendation:}

This paper reports on a improved CTH-based lightning scheme with the maritime lightning flash frequency being more realistic that the one of the PR92 lightning parameterization. The paper could be published in ACP but only after the authors have appropriately answered the questions and comments that I have addressed. There a number of points that need clarification and improvement before this manuscript can 
be accepted.

Please also note the supplement to this comment:

https://acp.copernicus.org/preprints/acp-2020-885/acp-2020-885-RC2-

supplement.pdf

Interactive comment on Atmos. Chem. Phys. Discuss., https://doi.org/10.5194/acp-2020-885, 2020. 